\title{
Quantitative Stress Measurements Near Blocked Slip Bands in Austenitic Stainless Steel Using High Resolution Electron Backscatter Diffraction.
}

\author{
D.C. Johnson ${ }^{1}$, M.D. McMurtrey ${ }^{2}$, G.S. Was ${ }^{1}$ \\ ${ }^{1 .}$ Dept. of Nuclear Engineering and Radiological Science, University of Michigan, Ann Arbor, MI \\ 48109 \\ 2. Dept. of Materials Science and Engineering, University of Virginia, Charlottesville, VA, 22904
}

The objective of this study is to quantitatively measure the stress profile ahead of a blocked slip band in an unirradiated austenitic stainless steel. Austenitic stainless steel is the main structural material for nuclear reactor core components and a potential material for advanced reactor concepts. Defect clusters, loops, and voids produced under irradiation change the deformation mode of these alloys from uniform slip to heterogeneous slip through dislocation channeling. When these dislocation channels interact with a grain boundary, the pile up of dislocations induces a stress at the head of the pile-up. It is believed that this stress increase is strongly correlated with stress corrosion cracking behavior. To date, no realistic quantitative measurements of this stress increase in stainless steels have been made [1].

As a precursor to quantitative measurement of localized stresses in irradiated samples, a method was developed for measuring the stress ahead of a blocked slip band in unirradiated material utilizing high resolution electron backscatter diffraction (HREBSD) patterns and a cross correlation software, CrossCourt3 (CC3), for data analysis. During cross correlation, a low stress reference pattern (taken from the same grain as analysis, but removed from high stress areas) is compared with every other pattern collected during the scan. Differences in the EBSD patterns can be used to generate a distortion tensor which is subsequently used to calculate the stress at each point. This technique was employed for stress measurements performed by Wilkinson et al [2] on a commercial purity titanium alloy. For this study, a Fe-21Cr-32Ni tensile bar was electropolished for EBSD analysis. A constant extension rate tensile (CERT) test was performed in an argon environment at $288^{\circ} \mathrm{C}$ to $0.7 \%$ total plastic strain. EBSD was then performed using a Philips XL30FEG and TSL 6 software.

Fig. 1 shows an area of the sample investigated where the slip bands are completely arrested at the grain boundary. This should correspond to the highest stress state since transmission of the slip band would relax some of the stress. The EBSD patterns taken from this area (Fig. 2) show high quality Kikuchi bands which are necessary for accurate stress analysis. Using CC3, the slip bands become visible when observing the geometric mean peak height map, Fig. 3. This plot is generated by correlating the intensity and rotation of each EBSD pattern to the reference with the value for auto correlation being set to 1 . Therefore the cross correlation technique is more sensitive to small changes in the pattern quality and can extract more information than image quality or inverse pole figure maps. The stress state as calculated by the CC3 software is shown in Fig 4. Visible here is plume of elevated stress values leading from the head of the slip band into the adjacent grain. The highest stress magnitude observed is at the interaction point, and drops significantly as the distance from the grain boundary increases. Stress values deviate from the matrix stress values over the first 2-3 microns. The magnitude of the stress for these measurements is qualitatively reasonable [3], with peak values in the range of 1-2 GPa for the von Mises stress. Less than $0.1 \%$ of the scan points had stress values greater than $10 \mathrm{GPa}$, but these points also had mean angular errors greater than $5 \times 10^{-3}$ radians and were removed from the analysis. Given the distribution of stress and the magnitude of measured values, HREBSD has been shown to be a plausible technique for analyzing unirradiated austenitic stainless steel. Similar analysis will be done in the future for irradiated samples to study the effects of irradiation on the stress state ahead of dislocation channels. 
References:

[1] MD McMurtrey et al, Plasticity 56 (2014), p. 219.

[2] TB Britton and AJ Wilkinson, Acta Materialia 60 (2012), p. 5773.

[3] TC Lee, IM Robertson, and HK Birnbaum, Philos Mag A 62 (1990), p. 131.

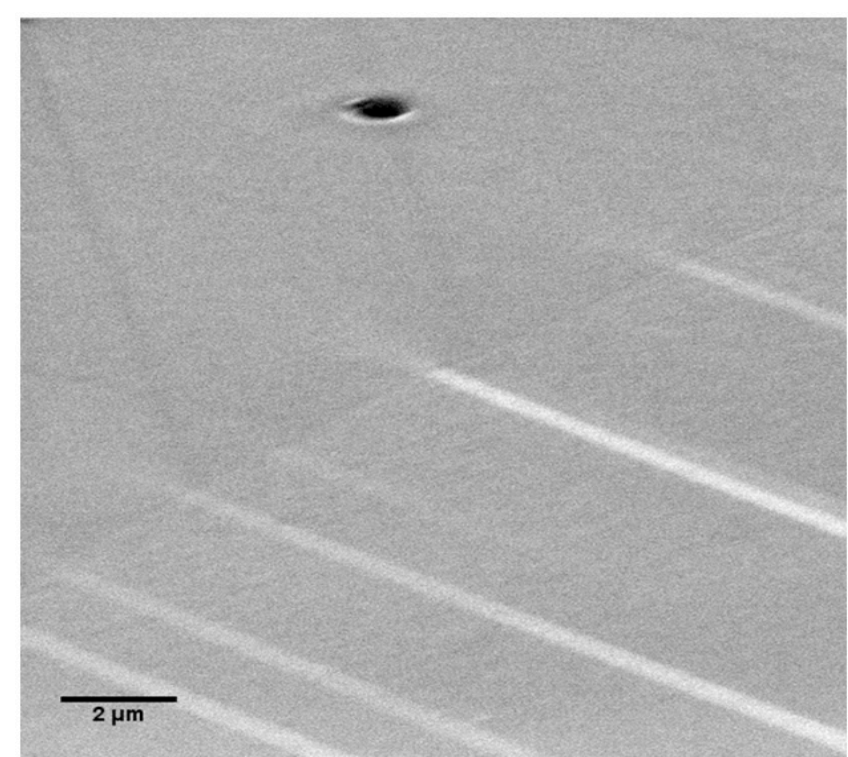

Figure 1: Slip band-grain boundary interaction

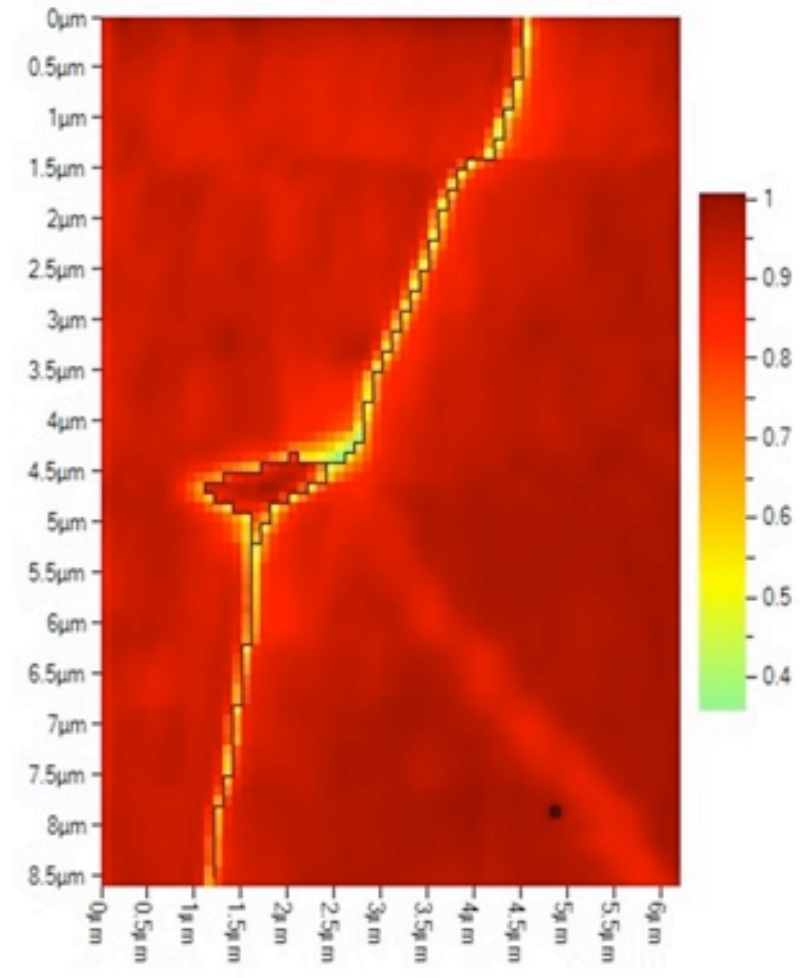

Figure 3: Geometric Mean Peak Height from CC3 analysis

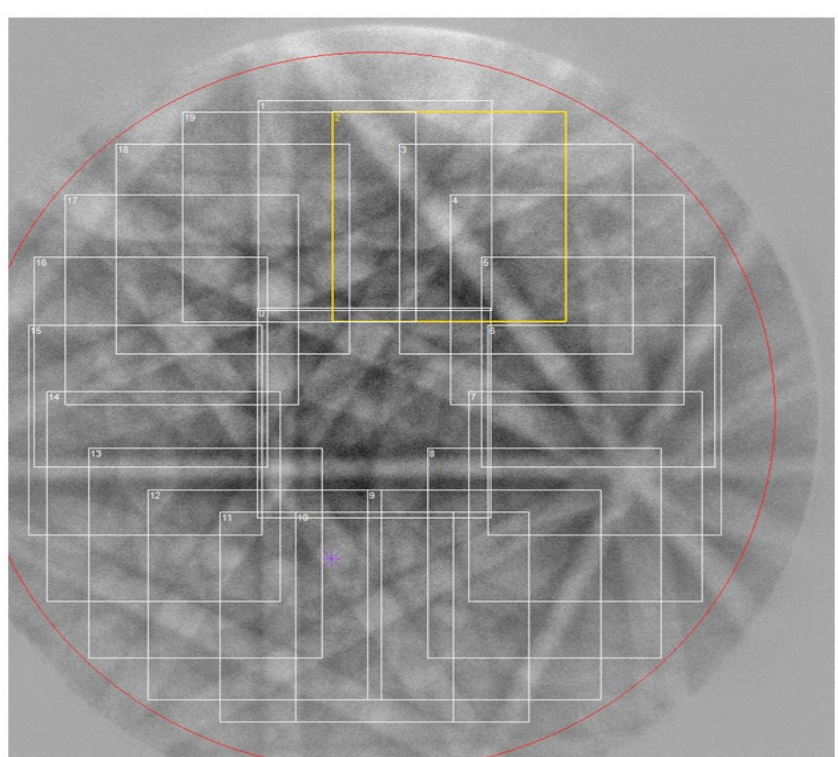

Figure 2: EBSD pattern taken from region in Fig. 1 for analysis in CC3

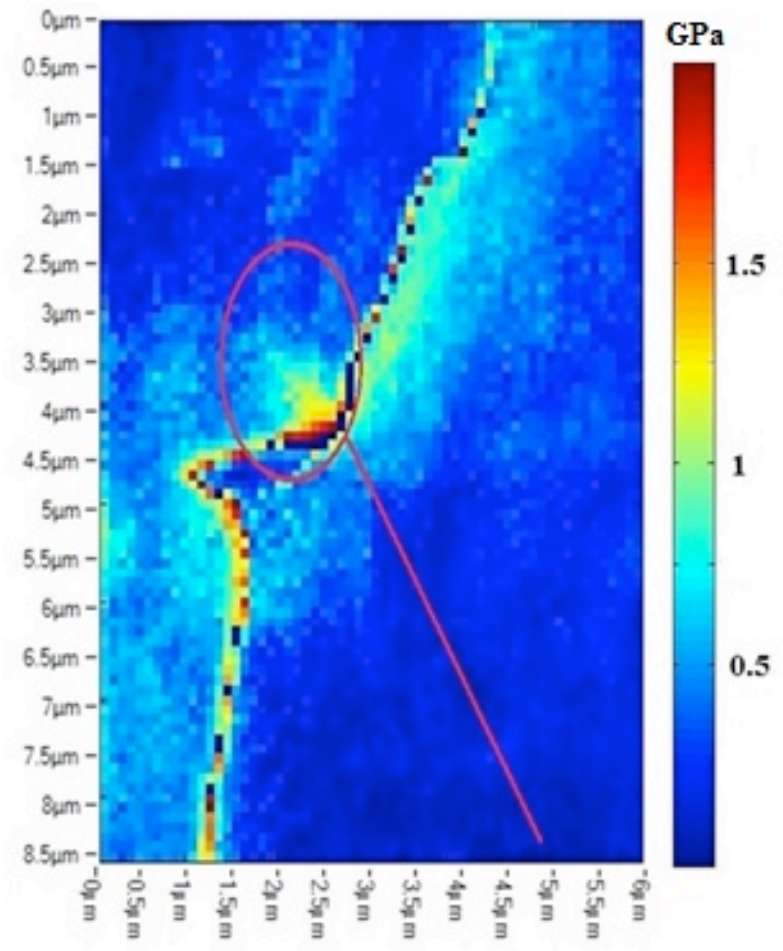

Figure 4: von Mises stress distribution at head of slip band 